\title{
Antiangiogenic therapy in breast cancer
}

\author{
Simon Peter Gampenrieder · Theresa Westphal · Richard Greil
}

Received: 12 September 2017 / Accepted: 23 October 2017 / Published online: 6 November 2017 (c) The Author(s) 2017. This article is an open access publication.

Summary Based on a strong rationale for antiVEGF (vascular endothelial growth factor) treatment in breast cancer and promising preclinical data, great hopes have been placed on the antiVEGF antibody bevacizumab. Clinical trials, however, reported conflicting results. In metastatic human epidermal growth factor receptor 2(HER2)-negative breast cancer, the addition of bevacizumab to standard chemotherapy improved consistently progression-free survival (PFS), however, without effect on overall survival (OS). In early breast cancer bevacizumab increased the pathologic complete response rate (pCR) after neoadjuvant therapy, but adjuvant trials did not demonstrate an effect on long-term survival. Unfortunately, despite extensive research, there is still no biomarker for bevacizumab efficacy available, making patient selection difficult. This review summarizes all phase III trials investigating efficacy and toxicity of bevacizumab in early, locally advanced and metastatic breast cancer. It recapitulates the main

\section{Authors' Contributions: Conception and design: Simon P. Gampenrieder; Collection and assembly of data: Simon P. Gampenrieder and Theresa Westphal; Data analysis and interpretation: All authors; Manuscript writing. Simon P. Gampenrieder and Theresa Westphal; Critical revising of the manuscript: Theresa Westphal, Richard Greil; Final approval of manuscript: All authors}

S. P. Gampenrieder · T. Westphal · R. Greil, MD (凶)

IIIrd Medical Department with Hematology and Medical Oncology, Oncologic Center, Paracelsus Medical University Salzburg, Müllner Hauptstraße 48, 5020 Salzburg, Austria r.greil@salk.at

S. P. Gampenrieder · T. Westphal · R. Greil, MD

Laboratory of Immunological and Molecular Cancer Research and Center for Clinical Cancer and Immunology Trials, Salzburg Cancer Research Institute, Salzburg, Austria

Cancer Cluster Salzburg, Salzburg, Austria toxicities, gives an overview on biomarker studies and discusses the role and future aspects of antiangiogenic therapy in breast cancer.

Keywords Breast cancer - Bevacizumab - Antiangiogenic therapy $\cdot$ Biomarker

\section{Introduction}

There is a strong rationale for the usage of antiangiogenic therapies in early, locally advanced and metastatic breast cancer. The concentration of hypoxia-inducible factor(HIF-1)alpha, a key player in angiogenesis regulation, is higher in breast tumors than in normal breast tissue and is even higher in poorly differentiated lesions than in the corresponding type of well-differentiated lesions [1]. Furthermore, increased angiogenesis, measured by vascular endothelial growth factor (VEGF) expression or microvessel density, is an independent negative prognostic factor in early breast cancer [2-4].

The addition of the anti-VEGF antibody bevacizumab to standard therapy improved both progression-free (PFS) and overall survival (OS) in several advanced cancers (colorectal cancer, non-small cell lung cancer, ovarian cancer, cervical cancer). In metastatic breast cancer (MBC), however, bevacizumab did not show an OS benefit and increased the grade $3 / 4$ toxicity rate. This led to a discussion about the clinical utility of this drug in this indication. In the neoadjuvant setting, several trials showed an improved pathologic complete response rate (pCR); however, adjuvant trials did not show any effect on disease-free (DFS) or OS. Other antiangiogenic drugs like sunitinib and sorafenib were investigated in advanced breast cancer as well, with consistently negative trial results $[5,6]$. Therefore, this review focuses on bevacizumab and summarizes the available clinical data in 
early, locally advanced and metastatic breast cancer, respectively.

\section{Bevacizumab in metastatic breast cancer}

\section{First-line trials}

Four prospective phase III trials investigated the efficacy and tolerability of bevacizumab in combination with chemotherapy as first-line therapy in human epidermal growth factor receptor 2(HER2)-negative metastatic breast cancer (Table 1). The approval for this indication was based on the results of the E2100 trial, where the combination of bevacizumab $10 \mathrm{mg} / \mathrm{kg}$ on days 1 and 15 and paclitaxel $90 \mathrm{mg} / \mathrm{m}^{2}$ on days 1,8 and 15 every 4 weeks showed a significant and clinically meaningful improvement in progression-free survival (PFS) compared to paclitaxel alone (11.8 months vs. 5.9 months; hazard ratio [HR] 0.60; $P<0.001$ ) [7]. The subsequent phase III trials (AVADO and RIBBON-1), however, found a much less pronounced effect on PFS with other chemotherapybackbones like docetaxel, capecitabine or anthracyclines [8, 9]. In AVADO the PFS difference was 1.9 months between docetaxel plus bevacizumab and docetaxel plus placebo [8]. In RIBBON-1 a PFS-difference of 2.9 months in the capecitabine cohort and of 1.2 months in the anthracycline/taxane cohort were reported ([9]; Table 1). The recently published MERiDiAN trial, using the same combination as in E2100, showed a similar median PFS of 11.0 months in the bevacizumab/paclitaxel arm compared to the E2100 trial. The control arm, however, performed much better with a median PFS of 8.8 months (5.6 months in E2100) resulting in a lower HR of $0.68(P<0.001$; Table 1). None of these trials, nor a subsumption in a meta-analysis, showed a benefit in OS by the addition of the anti-VEGF antibody and the rate of grade $3 / 4$ toxicity was increased $[10,11]$. In HER2-positive disease bevacizumab did not even show a significant improvement in PFS when added to docetaxel and trastuzumab (AVEREL trial, Table 1; [12]).

These results led in November 2011 to the decision of the Food and Drug Administration (FDA) to withdraw the accelerated approval for bevacizumab for the indication breast cancer. In contrast, based on the consistently improved PFS and overall response rate (ORR), bevacizumab is still approved in Europe by the EMA as first-line therapy in HER2-negative metastatic breast cancer when combined with paclitaxel or capecitabine. However, also in European countries like Austria, a significant decline in bevacizumab prescriptions for MBC became evident after the FDA decision [13].

The two approved chemotherapy backbones, each in combination with bevacizumab, were compared within the TURANDOT trial [14]. The primary objective of this study was to show non-inferiority of capecitabine/bevacizumab when compared to pa- clitaxel/bevacizumab in terms of OS. The primary endpoint, in fact, was met (median OS 30.2 months vs. 26.1 months; stratified HR 1.02; $P=0.007$ indicating non-inferiority); however, this was not supported by the unstratified analysis. In addition, the median PFS was significantly longer in the paclitaxel arm (10.9 months vs. 8.1 months 95\% CI 10.8-12.9; stratified HR 1.32, 95\%; $P=0.007$ ) and the objective response was also significantly superior with paclitaxel compared to capecitabine $(44 \%$ vs $27 \%$; $P<$ 0.001; [14]). In the ATHENA registry, including 2251 patients with HER2-negative breast cancer, the superiority of paclitaxel over capecitabine to improve time-to-progression (TTP: 9.8 months vs. 7.0 months) and response rate (49\% vs. $36 \%$ ) was confirmed (not statistically tested) [15].

Two phase III trials investigated the efficacy and tolerability of bevacizumab in combination with endocrine therapy for locally advanced or metastatic breast cancer. Both trials showed conflicting results: while the CALGB 40503 trial reported a statistically significant improvement in PFS by the addition of bevacizumab to letrozole (20.2 months vs. 15.6 months; HR 0.75; $P=0.016$ ) [16], the LEA trial failed to show superiority (median PFS 19.3 months vs. 14.4 months; HR $0.83, P=0.126$ ) [17]. In both trials, similar benefits were observed with respect to ORR and clinical benefit rate (CBR), but again neither study showed a difference in OS.

\section{Second-line trials}

Two phase III trials investigated bevacizumab after one prior chemotherapy-line for metastatic disease (RIBBON-2 and TANIA). Both trials showed an improvement in PFS by the addition of bevacizumab to different chemotherapy backbones ( $\triangle$ PFS 2.1 months in both trials; [18, 19]). Noteworthy, all patients included in the TANIA trial, where already pretreated with bevacizumab in the first-line setting and received bevacizumab for two additional therapy lines. Thus, the study investigated the principle of treatment-beyond-progression, as it is established in HER2-positive disease with trastuzumab. The primary endpoint, second-line PFS, was significantly longer when bevacizumab was continued (6.3 months vs. 4.2 months, HR $0.75 ; P=0.007$; [19]). The secondary endpoints, third-line PFS, second- plus third-line PFS and OS, however, where not improved ([20]; Table 1). The only chemotherapy-based phase III trial in HER2-negative MBC which did not show a significant improvement in PFS was the AVG2119g trial. In this pioneer trial, published already in 2005, patients pretreated with one or two chemotherapy lines for metastatic disease where randomized between bevacizumab plus capecitabine or capecitabine alone. Both the TANIA and the AVG2119g trial indicate that an early application of the anti-VEGF antibody is essential to gain the 


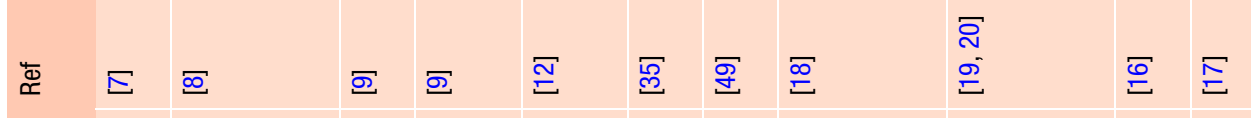

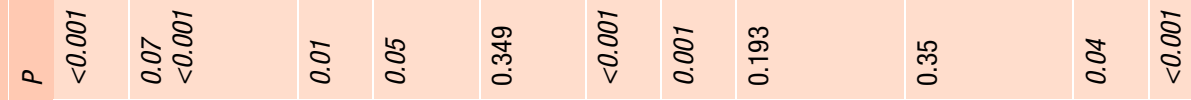

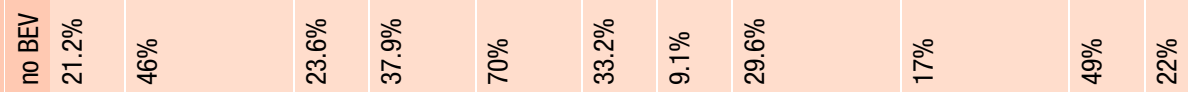

$\infty \$$

范 㟧

a

$\stackrel{\substack{0 \\ \stackrel{\pi}{3}}}{3}$

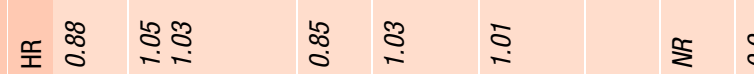

$\begin{array}{llll}m & 0 & 0 \\ 0 & 0 & 0 & 0\end{array}$

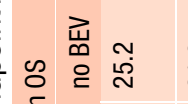

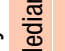

$\infty$

㟒

ڤ્ं

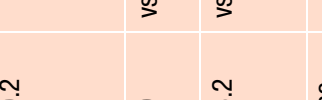

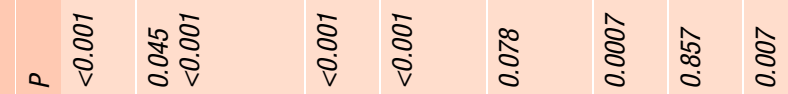

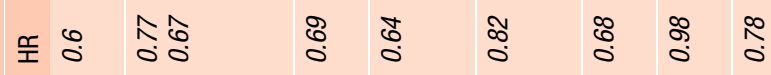

岕

ำ

i்

$\underset{\infty}{\infty} \stackrel{+}{\infty}$

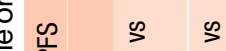

듬

㟒

$\infty$ ஸீ

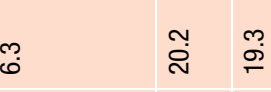

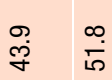

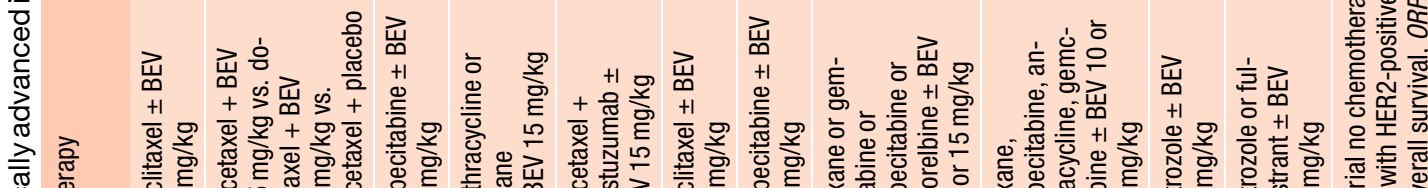

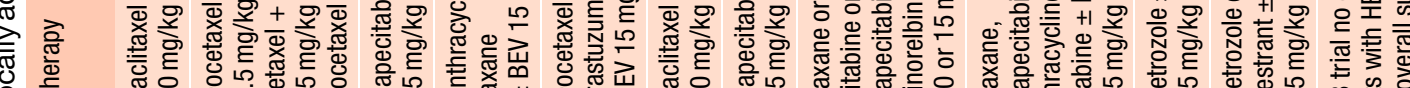

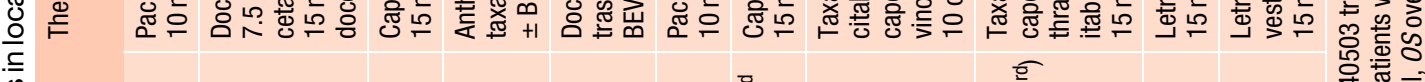

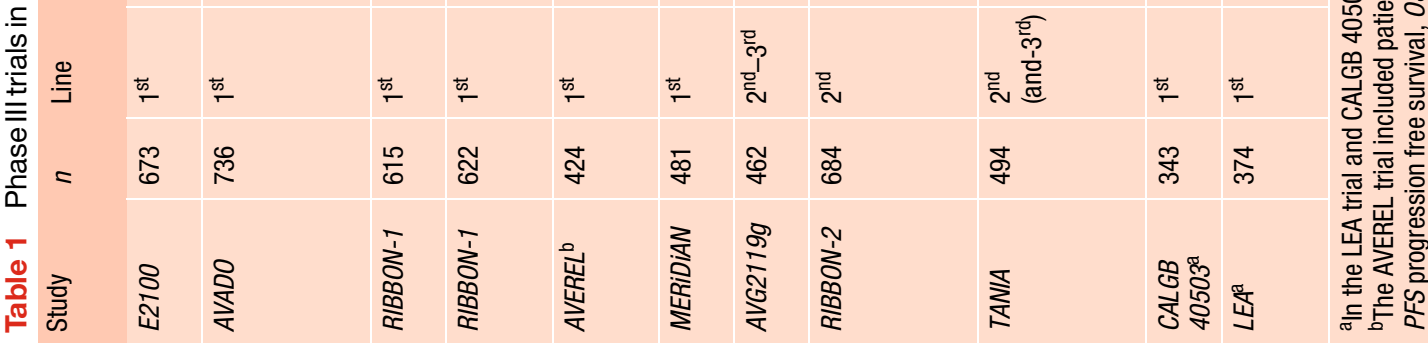


maximal benefit. Similar to the first-line trials, no trial beyond first-line showed an improvement in OS.

\section{Maintenance therapy}

One prospective trial addressed the question of maintenance therapy with bevacizumab after an induction phase with chemotherapy plus bevacizumab. Patients included in the IMELDA trial were treated with six cycles of docetaxel plus bevacizumab and were then randomized to maintenance therapy with bevacizumab alone or in combination with capecitabine. The combination therapy showed significantly improvement in PFS (median 11.9 months vs. 4.3 months; stratified HR 0.38 ; 95\%CI $0.27-0.55 ; P<0.0001 ;[21])$ indicating that maintenance bevacizumab alone is not an effective treatment option. Furthermore, the median duration of docetaxel induction was short (3.5 months) which may have contributed to the positive effect of early second-line chemotherapy with capecitabine.

Several retrospective case series investigated the question of maintenance therapy as well. A multicenter retrospective observational study, including 314 patients with HER2-negative disease, reported a significantly longer median PFS (18 months vs. 13 months; $P<0.001)$ and $\mathrm{OS}(55$ months vs. 38 months; $P<0.001$ ) in patients where maintenance therapy was prescribed [22]. These results, however, must be interpreted with caution because such retrospective non-randomized trials harbor the risk of multiple biases.

\section{Bevacizumab in early and locally advanced oper- able breast cancer}

\section{Neoadjuvant trials}

Based on the clear effect on response rate in the palliative setting, three phase III neoadjuvant trials with bevacizumab were started. All three trials showed a significantly higher rate of pathologic complete response (pCR) when bevacizumab was added to neoadjuvant chemotherapy (Table 2; [23-28]). The effect on pCR, however, was modest at best $(\triangle \mathrm{pCR} 5-11 \%)$ and no significant differences in DFS where reported. In the ARTEMIS trial, patients achieving a pCR in the bevacizumab group showed no longer DFS suggesting a lack of activity against micrometastases. Interestingly, the NSABP B-40 study, which additionally used bevacizumab postoperatively, showed a statistically significant difference in OS in favor of bevacizumab (HR 0.65; 95\%CI 0.49-0.88; $P=0.004$ ) [25]. In the context of the other neoadjuvant and adjuvant trials showing no effect on long-term survival; this observation should not be over-interpreted.

A special situation for neoadjuvant treatment is inflammatory breast cancer. Since this subtype is highly angiogenic showing high microvessel density and VEGF expression, an especially strong effect of bevacizumab was expected. A recent single arm phase II trial investigated FEC followed by docetaxel both in combination with bevacizumab every 3 weeks. Postoperatively, all patients received adjuvant bevacizumab (plus endocrine therapy in case of hormonereceptor positivity). The trial did not meet the prespecified criteria for efficacy with a pCR rate of $19 \%$ and a 3-year DFS of $57 \%(95 \% \mathrm{CI} 47-66 \%)$ and a median DFS of 53 months (95\%CI 31-not estimable); however, longer follow-up is needed for definitive conclusions [29].

\section{Adjuvant trials}

In the adjuvant setting three phase III trials were published until now (Table 3). The BEATRICE trial included patients with triple-negative disease [30, 31], the E5103 patients with HER2-negative [32] and the NSABP B44 patients with HER2-positive breast cancer [33]. The Kaplan-Meier curves for disease-free survival (DFS) and OS in all three were completely overlapping. This treatment failure can be explained by the fact that anti-angiogenic drugs can only work at a time point where neoangiogenesis is actually running, which is not the case in the adjuvant setting. Since senescent disease cannot be affected by antiangiogenic treatment and since no clinicopathological characteristics or biomarkers have been identified to indicate bevacizumab efficacy, anti-VEGF therapy has no application in the adjuvant setting.

\section{Toxicity}

In general, bevacizumab is a well-tolerated drug. The most frequent side effects when given as monotherapy are hypertension ( $15 \%$ grade $1 / 2,5 \%$ grade 3 ) and proteinuria ( $10 \%$ grade $1 / 2,2 \%$ grade 3 ), which are usually asymptomatic and manageable [31]. Infrequent but severe toxicities are left ventricular dysfunction $(\sim 1 \%)$, arterial thromboembolism including stroke or myocardial infarction $(<1 \%)$, major bleeding $(\sim 1 \%)$, wound-healing complications $(\sim 1 \%)$, osteonecrosis of the jaw $(<1 \%)$, gastrointestinal perforation or fistula $(<1 \%)$, reversible posterior leukoencephalopathy syndrome (RPLS; $<0.5 \%$ ), and infusion reactions $(<1 \%$; $[10,15,31])$. When added to chemotherapy, the incidences of neutropenia and febrile neutropenia are slightly increased (Table 4; [10]). These side effects, however, strongly depend on the type of chemotherapy backbone: while febrile neutropenia was low with weekly paclitaxel $(0.8 \%$ vs. $0 \%)$, the percentage was clearly higher with 3 -weekly docetaxel ( $16 \%$ vs. $11 \%)$ $[7,8]$. The same applies to sensory neuropathy with grade $3 / 4$ neuropathy in $24 \%$ vs. $18 \%$ of patients treated with paclitaxel and $3 \%$ vs. $0.5 \%$ of patients receiving capecitabine plus bevacizumab. The frequency of venous thrombotic events is not increased by the addition of bevacizumab to chemotherapy in patients with different metastatic tumors [34]. 


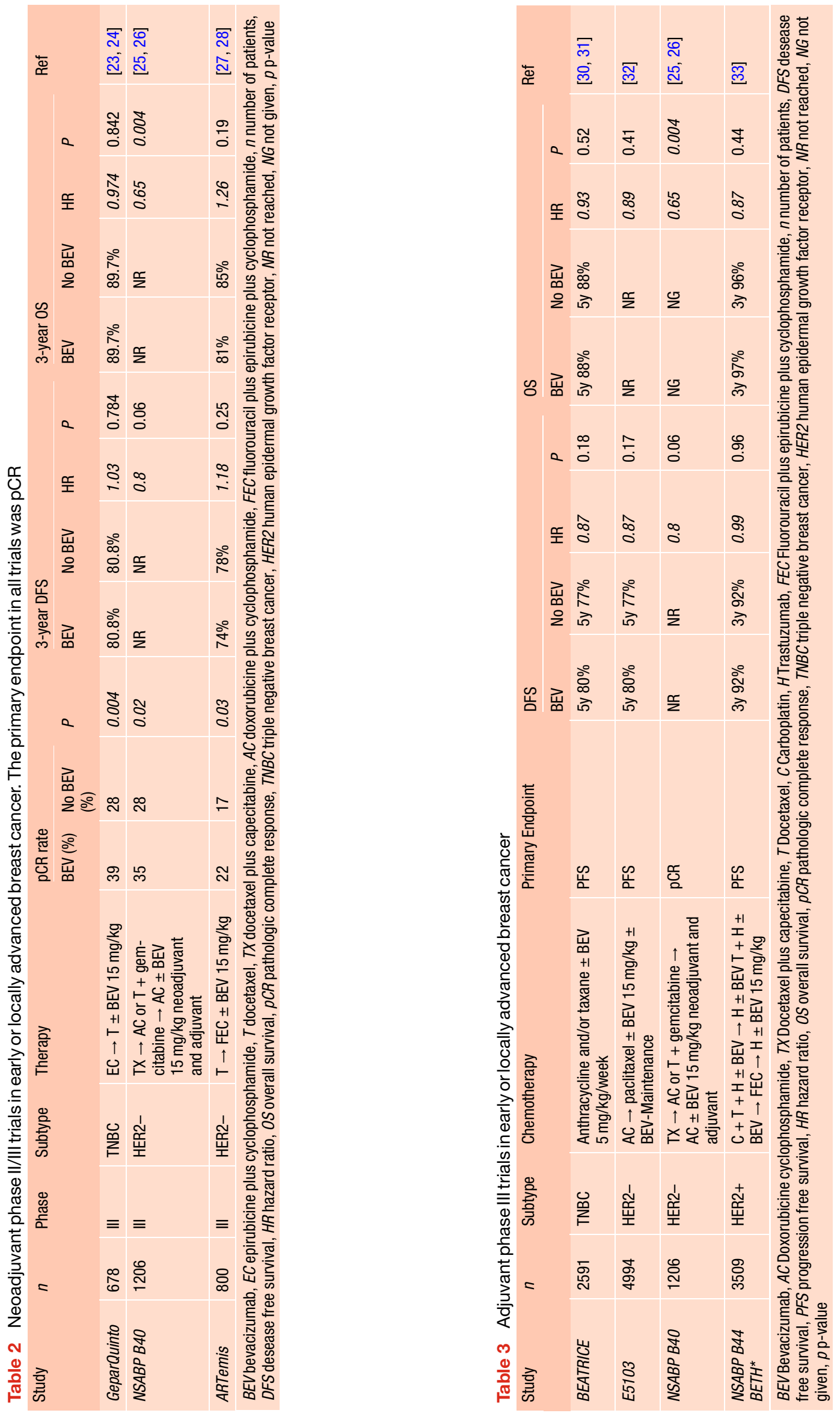


Table 4 Summary of selected grade $\geq 3$ adverse events of bevacizumab in combination with chemotherapy in three phase III trials in first-line [10]. Adverse events with a $>2 \%$ higher incidence in the bevacizumab group compared with the non-bevacizumab group are highlighted in italic

\begin{tabular}{|l|l|l}
\hline Grade 3/4 adverse event & $\begin{array}{l}\text { BEV (\%) } \\
(n=1679)\end{array}$ & $\begin{array}{l}\text { No BEV (\%) } \\
(n=982)\end{array}$ \\
\hline Neutropenia & 10.0 & 7.1 \\
\hline Sensory neuropathy & 9.5 & 8.5 \\
\hline Hypertension & 9.0 & 1.2 \\
\hline Febrile neutropenia & $6.5^{\mathrm{a}}$ & 3.5 \\
\hline Venous thromboembolic event & 2.8 & 3.8 \\
\hline Proteinuria & 2.3 & 0.0 \\
\hline Arterial thromboembolic event & 1.6 & 0.3 \\
\hline Bleeding & 1.5 & 0.4 \\
\hline Left ventricular systolic function & 1.5 & 0.2 \\
\hline Wound dehiscence & 0.8 & 0.3 \\
\hline Fistula & 0.5 & 0.3 \\
\hline Gl perforation & 0.5 & 0.3 \\
\hline RPLS & $<0.1$ & 0.0 \\
\hline
\end{tabular}

$B E V$ bevacizumab, Gl gastrointestinal, RPLS reversible posterior leukoencephalopathy syndrome, $T N B C$ triple-negative breast cancer ${ }^{\mathrm{a}} 0.8 \%$ with weekly paclitaxel in E2100 [7]

\section{Biomarker research}

The trials results, both in advanced and in early breast cancer, call for biomarkers allowing identification of patients with or without a relevant chance of clinical benefit from this drug. Unfortunately, clinicopathologic parameters like triple-negativity or highrisk features (visceral disease, $\geq 3$ metastatic sites, prior [neo]adjuvant chemotherapy) do not help to identify patients with special benefit from the addition of bevacizumab [10]. In a meta-analysis of the three firstline trials in metastatic breast cancer (E2100, AVADO, RIBBON-1), the hazard ratio (HR) for PFS in these subgroups was similar to the HR in the overall population ranging from 0.60 to 0.64 [10].

Several promising biomarkers like plasma levels of VEGF-A or VEGFR-2 [31, 35, 36], tissue markers like the VEGFR co-receptor neuropilin-1 (NRP-1) [37-39], single nucleotide polymorphisms (SNPs) in VEGF-A [40] or clinical markers like treatment-induced hypertension [40-42] showed convincing results in their early development as treatment predictors. However, all these markers either lack validating studies or failed to demonstrate clinical utility or reproducibility. The best example is plasma vascular endothelial growth factor-A (pVEGF-A), which showed a clear predictive value in the exploratory biomarker program of AVADO [36]. Therefore, the double-blind placebo-controlled randomized biomarker phase III trial MERiDiAN used the short isoforms of pVEGF-A as stratification factor [35]. Both co-primary endpoints of the trial were met: in the intent-to-treat (ITT) population, the stratified PFS HR was 0.68 (99\%CI $0.51-0.91 ; P<0.001)$ and in the VEGF-A $A_{\text {high }}$ subgroup, the stratified PFS HR was $0.64(96 \%$ CI $0.47-0.88 ; P=$ 0.004). The PFS benefit, however, was similar in the pVEGF-A $A_{\text {low }}$ subgroup (HR 0.73; 95\%CI 0.52-1.03). The VEGF-A-by-treatment interaction test $(P=0.462)$ for PFS in the ITT population did not support a predictive effect of pVEGF-A [35].

\section{Future aspects}

Despite extensive research, an applicable biomarker for bevacizumab efficacy is still lacking. Further research is ongoing; however, the patent expiry of Avastin ${ }^{\circledR}$ (Roche, Basel, Switzerland) in 2018 will probably hinder further development. For the same reason, no new phase III trials in breast cancer investigating solely the addition of bevacizumab to standard therapy are expected. However, besides its antiangiogenic activity, bevacizumab has a substantial immunomodulatory capacity. VEGF-A has been shown to suppress dendritic-cell maturation, to inhibit proliferation of regulatory $\mathrm{T}$ cells and to attract myeloid-derived suppressor cells [43-45]. These immunosuppressive effects are counteracted by bevacizumab. It has been shown that the tumor infiltration by CD4+ and CD8+ T cells and dendritic cells was increased after bevacizumab treatment and that the antigen-presenting capacity of dendritic cells was augmented [46-48]. These effects increase the immunogenicity of tumors and could therefore increase the number of patients benefiting from immunotherapy. Because of this potential synergistic effect, several combination studies with checkpoint inhibitors and bevacizumab are ongoing in different cancer types (colorectal cancer-NCT02982694, renal cell carcinoma-NCT02724878 and NCT01984242, melanoma-NCT03175432, cervical cancer-NCT029 21269, and non-small cell lung cancerNCT02366143). In case of positivity, the immunological effect of antiangiogenic therapeutics, which has been ignored for a long time, could lead to new treatment indications and new therapeutic goals, probably also in breast cancer.

Take home message Bevacizumab consistently prolonged progression-free survival and increased response rate in metastatic HER2-negative breast cancer in first- and second-line. An effect on overall survival, however, was not observed stirring up a debate about the value of bevacizumab in this indication. In the neoadjuvant setting the pathologic complete response rate (pCR) was increased, however in adjuvant trials no effect on disease-free survival was reported. Therefore, antiangiogenic treatment is not standard in early and locally advanced breast cancer at least until a biomarker for patient selection is available.

Funding Open access funding provided by Paracelsus Medical University. 
Conflict of interest Conflicts of interest with Roche ${ }^{\circledR}$ : Employment or Leadership Position: None; Consultant or Advisory Role: S.P. Gampenrieder, R. Greil; Stock Ownership: None; Speakers Honoraria: S.P. Gampenrieder, R. Greil; Travel Grants: S.P. Gampenrieder, T. Westphal, R. Greil; Research Funding: S.P. Gampenrieder, R. Greil.

Open Access This article is distributed under the terms of the Creative Commons Attribution 4.0 International License (http://creativecommons.org/licenses/by/4.0/), which permits unrestricted use, distribution, and reproduction in any medium, provided you give appropriate credit to the original author(s) and the source, provide a link to the Creative Commons license, and indicate if changes were made.

\section{References}

1. Bos $\mathrm{R}$, et al. Levels of hypoxia-inducible factor-1 alpha during breast carcinogenesis. J Natl Cancer Inst. 2001;93(4):309-14.

2. Uzzan B, et al. Microvessel density as a prognostic factor in women with breast cancer: a systematic review of the literature and meta-analysis. Cancer Res. 2004;64(9):2941-55.

3. Linderholm B, et al. Correlation of vascular endothelial growth factor content with recurrences, survival, and first relapse sitein primary node-positive breastcarcinoma after adjuvant treatment. JClin Oncol. 2000;18(7):1423-31.

4. Linderholm B, et al. Vascular endothelial growth factor is of high prognostic value in node-negative breast carcinoma. JClin Oncol. 1998;16(9):3121-8.

5. Bergh J, et al. First-line treatment of advanced breast cancer with sunitinib in combination with docetaxel versus docetaxel alone: results of a prospective, randomized phase III study. J Clin Oncol. 2012;30(9):921-9.

6. Baselga J, et al. RESILIENCE: phase III randomized, double-blind trial comparing Sorafenib with Capecitabine versus placebo with Capecitabine in locally advanced or metastatic HER2-negative breast cancer. Clin Breast Cancer. 2017; https://doi.org/10.1016/j.clbc.2017.05.006.

7. Miller K, et al. Paclitaxel plus bevacizumab versus paclitaxel alone for metastatic breast cancer. N Engl J Med. 2007;357(26):2666-76.

8. Miles DW, et al. Phase III study of bevacizumab plus docetaxel compared with placebo plus docetaxel for the first-line treatment of human epidermal growth factor receptor 2-negative metastatic breast cancer. J Clin Oncol. 2010;28(20):3239-47.

9. Robert NJ, et al. RIBBON-1: randomized, double-blind, placebo-controlled, phase III trial of chemotherapy with or without bevacizumab for first-line treatment of human epidermal growth factor receptor 2-negative, locally recurrent or metastatic breast cancer. J Clin Oncol. 2011;29(10):1252-60.

10. Miles DW, etal. First-line bevacizumab in combination with chemotherapy for HER2-negative metastatic breast cancer: pooled and subgroup analyses of data from 2447 patients. Ann Oncol. 2013;24(11):2773-80.

11. Rossari JR, et al. Bevacizumab and breast cancer: a meta-analysis of first-line phase III studies and a critical reappraisal of available evidence. J Oncol. 2012; https:// doi.org/10.1155/2012/417673.

12. Gianni L, et al. AVEREL: a randomized phase III trial evaluating Bevacizumab in combination with Docetaxel and Trastuzumab as first-line therapy for HER2-positive locally recurrent/metastatic breast cancer. J Clin Oncol. 2013;31(14):1719-25.
13. Preusser M, et al. Influence of the American ODAC statement on Austrian bevacizumab prescribing practice for metastatic breast cancer. Oncologist. 2012;17(7):e13-e7.

14. Zielinski C, et al. Bevacizumab plus paclitaxel versus bevacizumab plus capecitabine as first-line treatment for HER2-negative metastatic breast cancer (TURANDOT): primary endpoint results of a randomised, open-label, noninferiority, phase 3 trial. Lancet Oncol. 2016;17(9):1230-9.

15. Smith IE, et al. First-line bevacizumab plus taxane-based chemotherapy for locally recurrent or metastatic breast cancer: safety and efficacy in an open-label study in 2,251 patients. Ann Oncol. 2011;22(3):595-602.

16. Dickler MN, et al. Phase III trial evaluating Letrozole as first-line endocrine therapy with or without Bevacizumab for the treatment of postmenopausal women with hormone receptor-positive advanced-stage breast cancer: CALGB 40503 (alliance). JClin Oncol. 2016;34(22):2602-9.

17. Martin M, et al. Phase III trial evaluating the addition of bevacizumab to endocrine therapy as first-line treatment for advanced breast cancer: the letrozole/fulvestrant and avastin (LEA) study. JClin Oncol. 2015;33(9):1045-52.

18. Brufsky AM, et al. RIBBON-2: a randomized, double-blind, placebo-controlled, phase III trial evaluating the efficacy and safetyofbevacizumabin combination with chemotherapy for second-line treatment of human epidermal growth factor receptor 2-negative metastatic breast cancer. J Clin Oncol. 2011;29(32):4286-93.

19. von Minckwitz G, et al. Bevacizumab plus chemotherapy versus chemotherapy alone as second-line treatment for patients with HER2-negative locally recurrent or metastatic breast cancer after first-line treatment with bevacizumab plus chemotherapy (TANIA): an open-label, randomised phase 3 trial. Lancet Oncol. 2014;15(11):1269-78.

20. Vrdoljak E, et al. Final results of the TANIA randomised phase III trial of bevacizumab after progression on first-line bevacizumab therapy for HER2-negative locally recurrent/ metastatic breast cancer. Ann Oncol. 2016;27(11):2046-52.

21. Gligorov J, et al. Maintenance capecitabine and bevacizumab versus bevacizumab alone after initial first-line bevacizumab and docetaxel for patients with HER2-negative metastatic breast cancer (IMELDA): a randomised, open-label, phase 3 trial. Lancet Oncol. 2014; https://doi. org/10.1016/S1470-2045(14)70444-9.

22. Gamucci T, et al. A real-world multicentre retrospective study of Paclitaxel-Bevacizumab and maintenance therapy as first-line for HER2-negative metastatic breast cancer. JCell Physiol. 2017;232(6):1571-8.

23. Gerber B, et al. Neoadjuvant bevacizumab and anthracycline-taxane-based chemotherapy in 678 triple-negative primary breast cancers; results from the geparquinto study (GBG 44). Ann Oncol. 2013;24(12):2978-84.

24. von Minckwitz G, et al. Survival after neoadjuvant chemotherapy with or without bevacizumab or everolimus for HER2-negative primary breast cancer (GBG 44-GeparQuinto)dagger. Ann Oncol. 2014;25(12):2363-72.

25. Bear HD, et al. Neoadjuvant plus adjuvant bevacizumab in early breast cancer (NSABP B-40 [NRG Oncology]): secondary outcomes of a phase 3 , randomised controlled trial. Lancet Oncol. 2015;16(9):1037-48.

26. Bear HD, et al. Bevacizumab added to neoadjuvant chemotherapy for breast cancer. $\mathrm{N}$ Engl J Med. 2012;366(4):310-20.

27. Earl HM, et al. Disease-free and overall survival at 3.5 years for neoadjuvant bevacizumab added to docetaxel followed by fluorouracil, epirubicin and cyclophosphamide, for women with HER2 negative early breast cancer: ARTemis 
Trial. Ann Oncol. 2017; https://doi.org/10.1093/annonc/ mdxi73.

28. Earl HM, et al. Efficacy of neoadjuvant bevacizumab added to docetaxel followed by fluorouracil, epirubicin, and cyclophosphamide, for women with HER2-negative early breast cancer (ARTemis): an open-label, randomised, phase 3 trial. Lancet Oncol. 2015;16(6):656-66.

29. Bertucci F, et al. Bevacizumab plus neoadjuvant chemotherapy in patients with HER2-negative inflammatory breast cancer (BEVERLY-1): a multicentre, singlearm, phase 2 study. Lancet Oncol. 2016;17(5):600-11.

30. Bell R, et al. Final efficacy and updated safety results of the randomized phase III BEATRICE trial evaluating adjuvant bevacizumab-containing therapy in triple-negative early breast cancer. Ann Oncol. 2017;28(4):754-60.

31. Cameron D, et al. Adjuvant bevacizumab-containing therapy in triple-negative breast cancer (BEATRICE): primary results of a randomised, phase 3 trial. Lancet Oncol. 2013;14(10):933-42.

32. Miller K, et al. E5103 A double-blind phase III trial of Doxorubicin and Cyclophosphamidefollowed by Paclitaxel with Bevacizumab or placebo in patients with lymph node positive and high risk lymph node negative breast cancer. JClin Oncol. 2014;32:5.

33. Slamon DJ, et al. Primary results from BETH, a phase 3 controlled study of adjuvant chemotherapy and trastuzumab \pm bevacizumab in patients with HER2-positive, node-positive or high risk node-negative breast cancer. Cancer Res. 2013; https://doi.org/10.1158/0008-5472.sabcs13-s103. Abstract.

34. Hurwitz HI, et al. Venous thromboembolic events with chemotherapy plus bevacizumab: a pooled analysis of patients in randomized phase II and III studies. JClin Oncol. 2011;29(13):1757-64.

35. Miles D, et al. Bevacizumab plus paclitaxel versus placebo plus paclitaxel as first-line therapy for HER2negative metastatic breast cancer (MERiDiAN): a double-blind placebo-controlled randomised phase III trial with prospective biomarker evaluation. Eur J Cancer. 2017;70:146-55.

36. Miles DW, et al. Biomarker results from the AVADO phase 3 trial of first-line bevacizumab plus docetaxel for HER2negative metastatic breast cancer. Br J Cancer. 2013; https://doi.org/10.1038/bjc.2013.69.

37. Jubb AM, et al. Impact of exploratory biomarkers on the treatment effect of bevacizumab in metastatic breast cancer. Clin Cancer Res. 2011;17(2):372-81.

38. Van Cutsem E, et al. Bevacizumab in combination with chemotherapy as first-line therapy in advanced gastric cancer: a biomarker evaluation from the AVAGAST randomized phase III trial. JClin Oncol. 2012;30(17):2119-27.
39. Foernzler D, et al. Tumor tissue based biomarker analysis in NO16966: a randomized phase III study of firstline bevacizumab in combination with oxaliplatin-based chemotherapy in patients with mCRC. ASCO GI. 2012. abstr 374.

40. Schneider BP, et al. Association of vascular endothelial growth factor and vascular endothelial growth factor receptor-2 genetic polymorphisms with outcome in a trial of paclitaxel compared with paclitaxel plus bevacizumab in advanced breast cancer: ECOG 2100. J Clin Oncol. 2008;26(28):4672-8.

41. Hurwitz HI, et al. Analysis of early hypertension and clinical outcome with Bevacizumab: results from seven phase III studies. Oncologist. 2013;18(3):273-80.

42. GampenriederSP, etal. Hypertension as a predictivemarker for bevacizumab in metastatic breast cancer: results from a retrospective matched-pair analysis. Anticancer Res. 2014;34(1):227-33.

43. Terme M, et al. VEGFA-VEGFR pathway blockade inhibits tumor-induced regulatory $\mathrm{T}$-cell proliferation in colorectal cancer. Cancer Res. 2013;73(2):539-49.

44. Ohm JE, Carbone DP. VEGF as a mediator of tumor-associated immunodeficiency. Immunol Res. 2001;23(2-3):263-72.

45. Oyama T, et al. Vascular endothelial growth factor affects dendritic cell maturation through the inhibition of nuclear factor-kappa B activation in hemopoietic progenitor cells. J Immunol. 1998;160(3):1224-32.

46. Farsaci B, et al. Immune consequences of decreasing tumor vasculature with antiangiogenic tyrosine kinase inhibitors in combination with therapeutic vaccines. Cancer Immunol Res. 2014;2(11):1090-102.

47. Hodi FS, et al. Bevacizumab plus ipilimumab in patients with metastatic melanoma. Cancer Immunol Res. 2014;2(7):632-42.

48. Osada T, et al. The effect of anti-VEGF therapy on immature myeloid cell and dendritic cells in cancer patients. Cancer Immunol Immunother. 2008;57(8):1115-24.

49. Miller KD, et al. Randomized phase III trial of capecitabine compared with bevacizumab plus capecitabine in patients with previously treated metastatic breast cancer. J Clin Oncol. 2005;23(4):792-9.

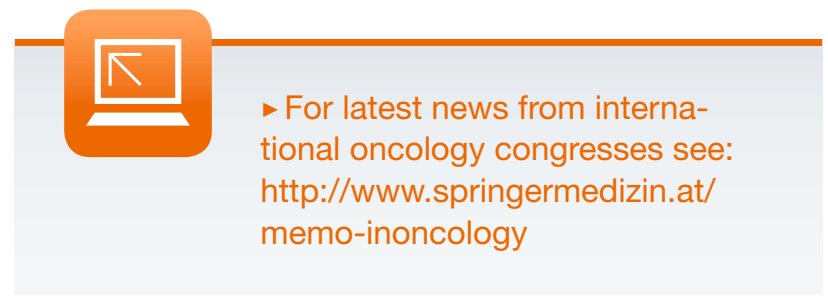

